\title{
Dynamic stability of a viscoelastic plate
}

\author{
Elena Kosheleva, ${ }^{1, *}$ \\ ${ }^{1}$ Moscow State University of Civil Engineering (National Research University) \\ Yaroslavskoe Shosse 26, Moscow, Russia
}

\begin{abstract}
The paper considers the problem of stability of a viscoelastic plate subjected to intermittent load. A differential equation was designed, which describes the dynamic stability of the plate stressed by permanent and alternating loads across the plate's plane. A solution in the form of a series with separated variables has been examined. Linear differential equations of the third order were obtained for the time function. A critical frequency equation was obtained as well. The major domains of dynamic instability were investigated. Another analysis was dedicated to the way the position of the prime instability domain is affected by relaxation time as well as correlation between long-term and instantaneous moduli of elasticity.
\end{abstract}

\section{Problem setting}

Let us consider a rectangular plate exposed to intermittent compressing forces across its plane, with these forces evenly distributed along the edges:

$$
N_{x}=N_{x 0}+N_{x t} \cos \theta t, \quad N_{y}=N_{y 0}+N_{y t} \cos \theta t
$$

The plate material is subject to a linear creep law, which is given by [1] for a flat strained state:

$$
\begin{gathered}
\sigma_{x}+n \dot{\sigma}_{x}=\frac{H}{1-v^{2}}\left(\varepsilon_{x}+v \varepsilon_{y}\right)+\frac{E n}{1-v^{2}}\left(\dot{\varepsilon}_{x}+v \dot{\varepsilon}_{y}\right), \\
\sigma_{y}+n \dot{\sigma}_{y}=\frac{H}{1-v^{2}}\left(\varepsilon_{y}+v \varepsilon_{x}\right)+\frac{E n}{1-v^{2}}\left(\dot{\varepsilon}_{y}+v \dot{\varepsilon}_{x}\right), \\
\tau_{x y}+n \dot{\tau}_{x y}=\frac{H}{2(1+v)} \gamma_{x y}+\frac{E n}{2(1+v)} \dot{\gamma}_{x y},
\end{gathered}
$$

where $n$-relaxation time, $E$ - instantaneous elasticity modulus, $H$ - long-term elasticitymodulus. Plate bending equation with (2) taken account of takes the form

$$
\frac{H}{E} D \nabla^{2} \nabla^{2} w+D n \frac{\partial}{\partial t} \nabla^{2} \nabla^{2} w=q+n \frac{\partial q}{\partial t},
$$

\footnotetext{
*Corresponding author: elleok@mail.ru
} 
where $D=\frac{E h^{3}}{\left(1-v^{2}\right)}$ - instantaneous bending rigidity. Load and inertial forces inserted into

(3) produce a differential equation of the third order:

$$
\begin{aligned}
& \frac{H}{E} D \nabla^{2} \nabla^{2} w+D n \frac{\partial}{\partial t} \nabla^{2} \nabla^{2} w+m \frac{\partial^{2} w}{\partial t^{2}}+n m \frac{\partial^{3} w}{\partial t^{3}}+N_{x} \frac{\partial^{2} w}{\partial x^{2}}+ \\
& N_{y} \frac{\partial^{2} w}{\partial y^{2}}+n\left(\frac{\partial N_{x}}{\partial t} \frac{\partial^{2} w}{\partial x^{2}}+\frac{\partial^{3} w}{\partial x^{2} \partial t} N_{x}+\frac{\partial N_{y}}{\partial t} \frac{\partial^{2} w}{\partial y^{2}}+\frac{\partial^{3} w}{\partial y^{2} \partial t} N_{y}\right)=0 .
\end{aligned}
$$

\section{Solution technique}

The solution to this equation can be found by separating the variables:

$$
w=\sum f_{i}(t) X_{i}(x) Y_{i}(y)
$$

where $f_{i}(t)$ - the unknown time functions, $X_{i}(x)$ and $Y_{i}(y)$ - functions fulfilling the boundary conditions along the edges of the rectangular outline relative to $w$. By inserting the solution (5) to (4) and by utilizing the Bubnov-Galerkin method, an equation with alternating coefficients is obtained:

$$
f^{\prime \prime \prime}+\frac{1}{n \theta} f^{\prime \prime}+\frac{\Omega^{2}}{\theta^{2}}(1-2 \mu \cos \tau) f^{\prime}+\frac{\Omega^{2}}{n \theta^{2}}(\xi-2 \mu \cos \tau+2 \mu n \theta \sin \tau) f=0,
$$

where the prime denotes a nondimensional time derivative $\tau=\theta t, \Omega-$ proper vibration frequency of the plate exposed to the constant components of longitudinal forces, $\mu$ excitation coefficient, $\xi$ - nondimensional parameter determined by the correlation between the instantaneous and long-haul elasticity modulus;

$$
\begin{gathered}
\Omega^{2}=\omega^{2}\left(1-\frac{N_{x 0} N_{2 *}+N_{y 0} N_{1 *}}{N_{1 *} N_{2 *}}\right), \\
\mu=\frac{1}{2} \frac{N_{x t} N_{2 *}+N_{y t} N_{1 *}}{N_{1 *} N_{2 *}-N_{x 0} N_{2 *}-N_{y 0} N_{1 *}}, \xi=\frac{\frac{H}{E} N_{1 *} N_{2 *}-N_{x 0} N_{2 *}-N_{y 0} N_{1 *}}{N_{1 *} N_{2 *}-N_{x 0} N_{2 *}-N_{y 0} N_{1 *}},
\end{gathered}
$$

$N_{1 *}$ и $N_{2 *}$ - critical values of strains $N_{x 0}$ and $N_{y 0}$ provided that their statical action is independent, $\omega$-frequency of natural vibration of an unloaded plate,

$$
\begin{aligned}
& \omega^{2}=\frac{D I_{1}}{m I_{2}}, N_{1 *}=\frac{I_{1}}{I_{3}} D, N_{2 *}=\frac{I_{1}}{I_{4}} D, I_{1}=\int_{0}^{a} \int_{0}^{b}\left(\frac{d^{4} X}{d x^{4}} Y+2 \frac{d^{2} X}{d x^{2}} \frac{d Y^{2}}{d y^{2}}+\frac{d Y^{4}}{d y^{4}} X\right) X Y d x d y, \\
& I_{2}=\int_{0}^{a} \int_{0}^{b} X^{2} Y^{2} d x d y, I_{3}=-\int_{0}^{a} \int_{0}^{b} X \frac{d^{2} X}{d x^{2}} Y^{2} d x d y, I_{4}=-\int_{0}^{b} \int_{0}^{b} X^{2} \frac{d^{2} Y}{d y^{2}} Y d x d y
\end{aligned}
$$

The equation features four nondimensional coefficients $\mu, \xi, \quad \alpha=\frac{1}{n \theta}, \varphi=\frac{\Omega^{2}}{\theta^{2}}$. Changes in these coefficients impact the solution to the equation (6). Provided certain correlations between the coefficients are in place, this equation may have infinitely 
increasing solutions, which take up entire domains on the parameter plane $\mu, \frac{\theta}{2 \Omega}$. Defining locations of dynamic instability domains represents one of the major tasks of the dynamic stability theory. The domains of boundlessly increasing solutions are separated from the domains of stability by intermittent solutions at intervals $T$ and $2 T$. Two solutions of the same period limit the domain of instability, two solutions of different periods do same for the domain of stability. The search for instability domains comes down to determination of conditions under which the equation (6) has periodic solutions with the period being $T$ and $2 T$. The solution to the equation (6) with the period $T$ is searched for in the form of a seriesse

$$
f(t)=\sum_{k=1,3,5}^{\infty}\left(a_{k} \sin \frac{k \tau}{2}+b_{k} \cos \frac{k \tau}{2}\right),
$$

while the solution with the $2 T$ period is searched for in the form of a series

$$
f(t)=\sum_{k=2,4,6}^{\infty}\left(a_{k} \sin \frac{k \tau}{2}+b_{k} \cos \frac{k \tau}{2}\right)+b_{0} .
$$

The first region of instability is of paramount importance, hence we insert the solution (9) into the equation (6) :

$$
\begin{aligned}
& \sum_{k=1,3}^{\infty}\left(-a_{k} \frac{k^{8}}{8} \sin \frac{k \tau}{2}+b_{k} \frac{k^{3}}{8} \cos \frac{k \tau}{2}\right)+\alpha \sum_{k=1,3}^{\infty}\left(-a_{k} \frac{k^{2}}{4} \sin \frac{k \tau}{2}-b_{k} \frac{k^{2}}{4} \cos \frac{k \tau}{2}\right)+ \\
& \varphi\left(1-2 \mu \cos \tau+\frac{2 \mu}{\alpha} \sin \tau\right) \sum_{1,3}^{\infty}\left(a_{k} \sin \frac{k \tau}{2}+b_{k} \cos \frac{k \tau}{2}\right)=0 .
\end{aligned}
$$

After transforming the equation (11) and equalizing the coefficiens, $\sin \frac{k \tau}{2}$ and $\cos \frac{k \tau}{2}$ being identical, we obtain a system of linear equations in respect of $a_{k}$ and $b_{k}$ :

$$
\begin{aligned}
& a_{1} \alpha\left(-\frac{1}{4}+\varphi \xi+\varphi \mu\right)-\alpha \varphi \mu a_{3}-\frac{1}{2} b_{1}\left(-\frac{1}{4}+\varphi-\varphi \mu\right)+\frac{1}{2} \varphi \mu b_{3}=0 \\
& \frac{1}{2} a_{1}\left(-\frac{1}{4}+\varphi+\varphi \mu\right)+\alpha b_{1}\left(-\frac{1}{4}+\varphi \xi-\varphi \mu\right)-\frac{1}{2} \varphi \mu a_{3}-\alpha \varphi \mu b_{3}=0 \\
& \alpha\left(-\frac{k^{2}}{4}+\varphi \xi\right) a_{k}-\frac{k}{2} b_{k}\left(-\frac{k^{2}}{4}+\varphi\right)-\alpha \varphi \mu\left(a_{k+2}+a_{k-2}\right)+\varphi \mu \frac{k}{2}\left(b_{k+2}-b_{k-2}\right)=0 \\
& \frac{k}{2} a_{k}\left(-\frac{k^{2}}{4}+\varphi\right)+\alpha b_{k}\left(-\frac{k^{2}}{4}+\varphi \xi\right)-\varphi \mu \frac{k}{2}\left(a_{k+2}+a_{k-2}\right)-\alpha \varphi \mu\left(b_{k+2}+b_{k-2}\right)=0, \\
& (k=1,3,5 \ldots)
\end{aligned}
$$

An equation of critical frequencies is obtained by setting the equation determinant to zero.

By critical frequencies we understand the frequencies of the external load $\theta_{*}$, corresponding to the boundaries of the instability domains. By substituting the coefficient 
$\varphi$ in (12) by $p^{2}=\frac{1}{4 \varphi}$, we transform the coefficient $\alpha=\frac{1}{n \theta}=\frac{r}{p}$, where $\quad r=\frac{1}{2 \Omega n}$. Consequently, we obtain

$$
\left|\begin{array}{ccccc}
\frac{r}{p}\left(-p^{2}+\xi+\mu\right) & -\frac{1}{2}\left(-p^{2}+1-\mu\right) & -\frac{r}{p} \mu & \frac{1}{2} \mu & . \\
\frac{1}{2}\left(-p^{2}+1+\mu\right) & \frac{r}{p}\left(-p^{2}+\xi-\mu\right) & -\frac{1}{2} \mu & -\frac{r}{p} \mu & . \\
-\frac{r}{p} \mu & \frac{3}{2} \mu & \frac{r}{p}\left(-9 p^{2}+\xi\right) & -\frac{3}{2}\left(-9 p^{2}+1\right) & . . \\
-\frac{3}{2} \mu & -\frac{r}{p} \mu & \frac{3}{2}\left(-9 p^{2}+1\right) & \frac{r}{p}\left(-9 p^{2}+\xi\right) & \ldots \\
\ldots & \ldots & \ldots & \ldots
\end{array}\right|=0 .
$$

This equation facilitates finding the domains of dynamic instability which lie in the proximity of the frequencies $\theta_{*}=\frac{2 \Omega}{k}, k=1,3,5 \ldots \quad$ Next we are going to consider the principal region of instability in the proximity of $\theta_{*}=2 \Omega$, which is the most dangerous and for this reason has the most significant practical implications.

\section{The findings}

Three iterations were made to build the main instability domain, i.e. determinants of the second, fourth and sixth order were considered. The second and third iterations yielded close results, and for this reason the determinant of the fourth order was used to investigate the instability domain. The equation (13) encompasses coefficients $r$ and $\xi$, one of which depends on the relaxation time, while the other hinges on the correlation between the instantaneous and long-term moduli of elasticity. Changes in these coeffictions impact the

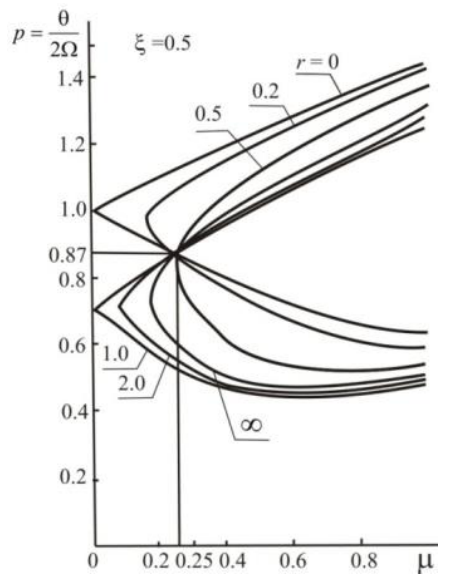

Figure 1 Major areas of sustainability position of the instability domains. To analyze the impact of the coefficients we are going to build the domains of dynamic instability where $\xi$ is constant and specific while $r$ is variable. Figure 1 displays the domains for the value $\xi=0.5$, while $r$ assumes values from zero to infinitely high values. The figure makes it clear that with $r$ increasing, the boundaries of the domain initially depart from the $p$ axis and move to the right, and at the same time displace vertically towards the reduction of $p$, and then approach the axis again.

The instability domain with $r=0$ begins from the point, the coordinate of which is $p=1$, while the domain stems from the point with coordinates $p=\sqrt{\xi}=0.707$ given $r=\infty$. With $r=0$, which implies infinitely long relaxation time, the material showcases elasticity with an instantaneous elasticity modulus $E$. In constrast, $r=\infty$, which corresponds to $n=0$, makes the material perform with elasticity, $H$ being the respective long-haul elasticity modulus. Qualitative similarity may be noticed if a 
comparison is drawn between the outcome displayed in Figure 1 and the borders of the domains built for a beam [3]. In what follows we consider the dependency between $\xi$ and $r$. Figure 2 presents the results of such calculations. The impact of $\xi$ on the position of the domain depends, as the graphs indicate, on the magnitude of $r$.

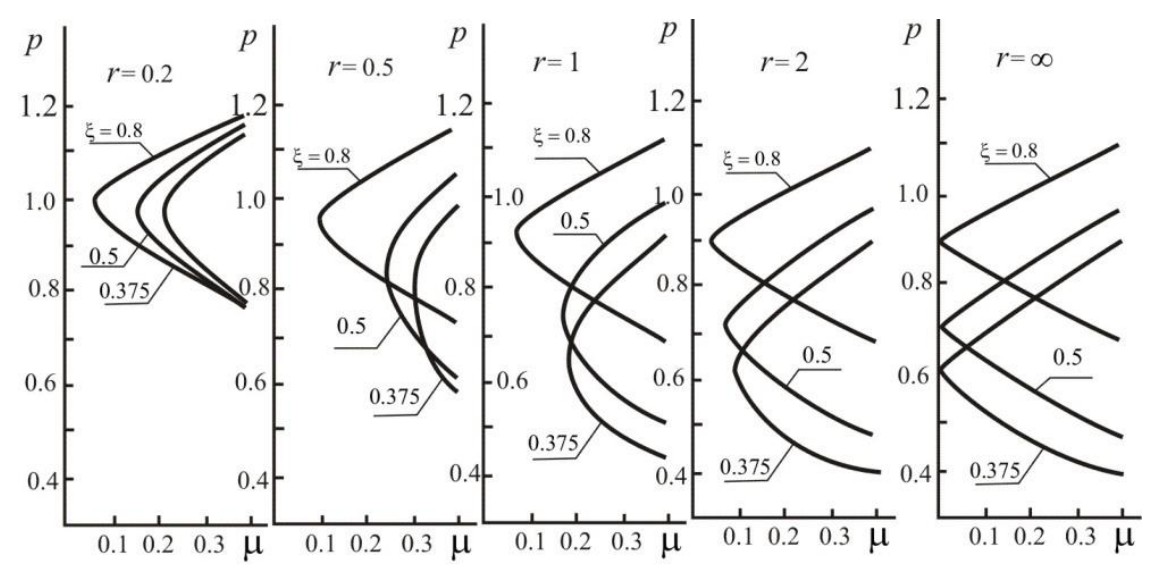

Figure 2 Influence of $\xi$ on the principal domains of dynamic stability, depending on the value of $r$

Decrease in $\xi$ with $r$ being constant, causes the boundary to displace towards the increasing $\mu$. With $r$ increasing, such displacement reduces, while $r=\infty$ means that all the curves border upon the $p$ axis. At the same time, increasing $r$ combined with decreasing $\xi$ displaces the curves towards the $\mu$ axis. The parameter $\xi$ depends on the correlation of the elasticity moduli; if this parameter goes down against the backdrop of the constant relaxation rate (unless it is equal to zero or is infinitely high), the minimum value of the excitation coefficient $\mu$ increases, and thus the minimum amplitudes of periodic forces $N_{x t}$ and $N_{y t}$ rise as well, while the frequency $\theta$ goes down. If the relaxation rate is equal to zero, the material displays elastic behavior, $E$ representing the instantaneous elastic modulus. If the relaxation rate assumes an infinitely high value, decreasing $\xi$ is accompanied by the lowering load frequency, which leads to stability being lost.

We thus have investigated the way the parameter $\xi$ determines the position of the boundaries of the main instability domain. In case the correlation between the elasticity moduli narrows down, and hence $\xi$ decreases when the relaxation rate is constant, unless it is zero or infinitely high, the minimum value of the excitation coefficient as well as the minimum amplitudes of periodic forces increase, while the frequencies of these forces contract. Infinitely high speed of relaxation along with reduction of the correlation in question causes a fall in load frequency, which accounts for a loss of dynamic stability.

\section{Conclusion}

The paper has explored the way relaxation time and correlation between elasticity moduli impact the position of the principal dynamic instability domain. It has been demonstrated that the lower the correlation between the elasticity moduli with relaxation speed being steady (except for its zero and infinitely high values), the higher the minimum 
value of the excitation coefficient, the higher the minimum amplitudes of periodic forces, while the frequency of these forces goes down. Viscoelastic behavior of structural elements is most relevant for polymers and composite materials 0[4]. Recent time has seen multiple papers exhibiting new results of research dedicated to viscoelastic characteristics of materials and development of computing techniques for respective structures [5-9 etc.]

\section{References}

1. V.V. Bolotin, Dynamic Stability of Elastic Systems (State publishing house of theoretic technical literature, 1956)

2. A.R. Rzhanitsyn, Creep theory. (Stroyizdat M., 1968)

3. V.I. Matyash, Mechanics of polymers 1, p.116-121(1974)

4. R.A. Turusov, V.I. Andreyev, B.M. Yaziyev, Selected Problems and Techniques of Mechanics of Macroheterogenous Viscoelastic Environment. (RGSU Publishing, Rostov-on-Don ,2009)

5. N.Yu.Tsybin, R.A.Turusov, V.I.Andreev, Comparison of creep in free polymer rod and creep in polymer layer of the layered composite. Procedia engineering 153 ( 2016 ) $51-58$

6. V.I. Andreyev, B.M. Yaziyev, Buckling of Lengthwise Clamped Bars of Alternating Rigidity under Creep. Inzhenerny Vestnik Dona (Engineering Reporter of Rostov-on-Don) 4 (2012) 142-148.

7. V.I. Andreev, A.S. Chepurnenko, B.M. Yazyev, On the Bending of a Thin Plate at Nonlinear Creep. Advanced Materials Research, 900 (2014) 707-710

8. V.I. Andreev, A.S. Chepurnenko, B.M. Yazyev, Energy Method in the Calculation Stability of Compressed Polymer Rods Considering Creep. Advanced Materials Research 1004-1005 (2014) 257-260

9. V.I. Andreev, A.S. Chepurnenko, B.M. Yazyev, A.N. Beskopylny Determination of Rheological Parameters of Polyvinylchloride at Different Temperatures. MATEC Web of Conferences 67 (2016) No 06059 